\title{
Predictive Soft Error Rate Evaluation System
}

\author{
Phil Oldiges, Brett Knowlton ${ }^{a}$ and Robert Flatley \\ Digital Equipment Corporation, Hudson, MA, USA \\ aNow with Unica Technologies, Lexington, MA, USA
}

\begin{abstract}
A set of simulation tools lias been developed to model the effects of alphaparticles and cosmic ray neutrons on various circuit elements. Two tools have been written to evaluate the effects of alpha-particles on circuits. The first tool uses monte carlo integration techniques and calibrated computationally efficient charge collection models to allow for fast estimates of charge collection and soft error rate. The second tool determines the effects of alpha-particles emitted from solder bumps on underlying circuits due to the specific geometry of the solder bump. A final simulation tool uses deterministic methods to integrate over point burst rate for determining cosmic ray neutron induced soft error rates. Comparisons with measurements as well as descriptions of the models are discussed for all of the simulation tools.
\end{abstract}

\section{Introduction}

Soft errors occur in circuits as a result of energetic particles striking the silicon substrate and generating charge near reverse-biased junctions [1]. The particles that cause the majority of problems in circuits are $\alpha$-particles and cosmic rays $[2,3] . \alpha-$ Particles can come from many sources, but the sources that generate the majority of $\alpha$-particles affecting circuits are package materials, metal layers, chemicals used in processing and other sundry materials used in IC manufacturing [4]. The cosmic ray that causes the most serious soft error problem in circuits at sea level or low altitudes is neutrons [5]. A simulation system has been developed to aid in the understanding of the effects of neutrons and $\alpha$-particles in circuits.

\section{2. $\alpha$-Particle Induced Soft Error Simulation}

Recent enhancements have been made to the Soft Error EValuator, SEEV [6]. SEEV uses Monte Carlo techniques to integrate over all of the possible $\alpha$-particle energies and angles emitted from a particular $\alpha$-particle source. Computationally efficient charge collection models are incorporated into the simulator to allow for fast estimates of charge collection and soft error rate [6]. Recently, measurements have been performed of the soft error rate on a $0.25 \mu \mathrm{m}$ SRAM as a function of the incidence angle of $\alpha$ particles. This data was used to calibrate the $\alpha$ particle incidence angle dependent charge collection model used in SEEV. Figure 1 shows a comparison of the measurement and calculated soft error rate. SEEV shows an excellent fit to the measurements. 


\section{Simulated and measured angular dependence of SER}

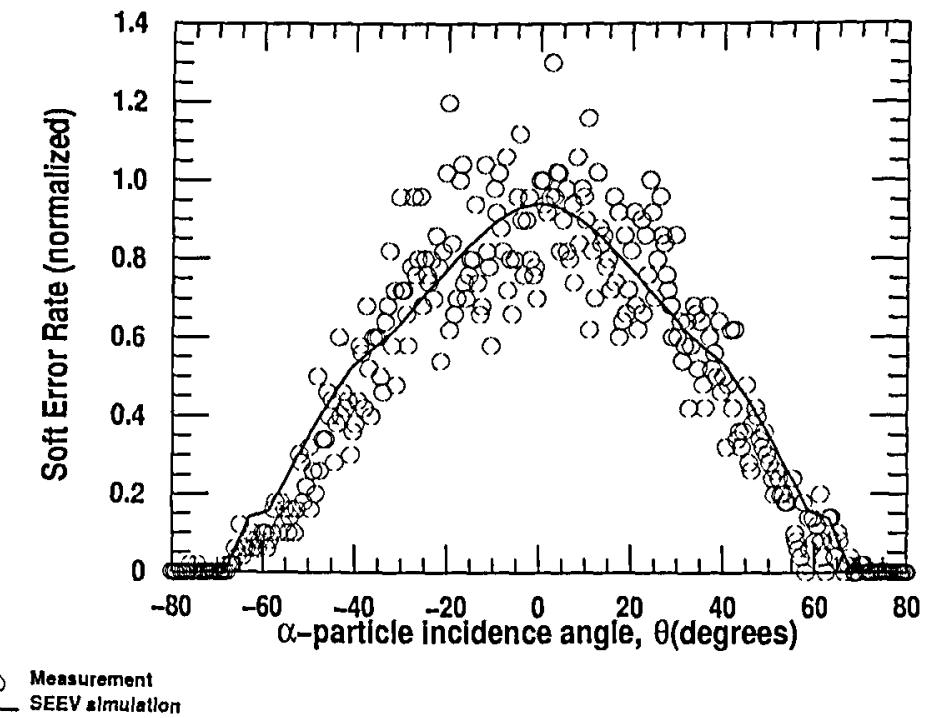

Figure 1: Comparison of simulated and measured soft error rate for a $0.25 \mu \mathrm{m}$ SRAM. The $\alpha$-particle incidence angle is measured from normal incidence.

As the number of $\mathrm{I} / \mathrm{O}$ pads increases, it is important to be able to get signals into and out of the circuit as efficiently as possible. Flip-chip technology is an option being considered to allow for large scale I/O and power routing. Since solder is a notoriously "dirty" material, emitting a large $\alpha$-particle flux, SEEV was modified to simulate the effects of $\alpha$-particles emitted from solder bumps. Figure 2 shows the basic geometry of the structure that can be simulated. On top of a silicon substrate is a dielectric layer. On top of that, a solder bump is defined. We also allow for a filler material to be placed adjacent to the solder bump. We assume cylindrical symmetry, but the solder bump can be defined in any arbitrary shape. Monte Carlo techniques allow $\alpha$-particles to be emitted from the solder ball in arbitrary directions. Each $\alpha$-particle is tracked through the various materials until it strikes the silicon surface. Figure 3 shows a typical result that can be obtained from this simulation. Figure 3 shows the radial dependence of $\alpha$-particle flux at the silicon surface. This example shows the importance of having an underfill material directly adjacent to the solder bump. The underfill material stops $\alpha$-particles that would otherwise affect circuits a large distance away from the solder bump.

\section{Neutron Induced Soft Error Simulation}

Since there is a large flux of energetic neutrons even at sea level, the soft error rate of a circuit can be dominated by cosmic ray strikes. Simulations of the neutron induced soft error rate are now able to be performed. Figure 4 shows the results of calculations compared to measurements made on $0.25 \mu \mathrm{m}$ SRAMs. The agreement with measurements is excellent. Unlike the $\alpha$-particle soft error rate simulation, this 


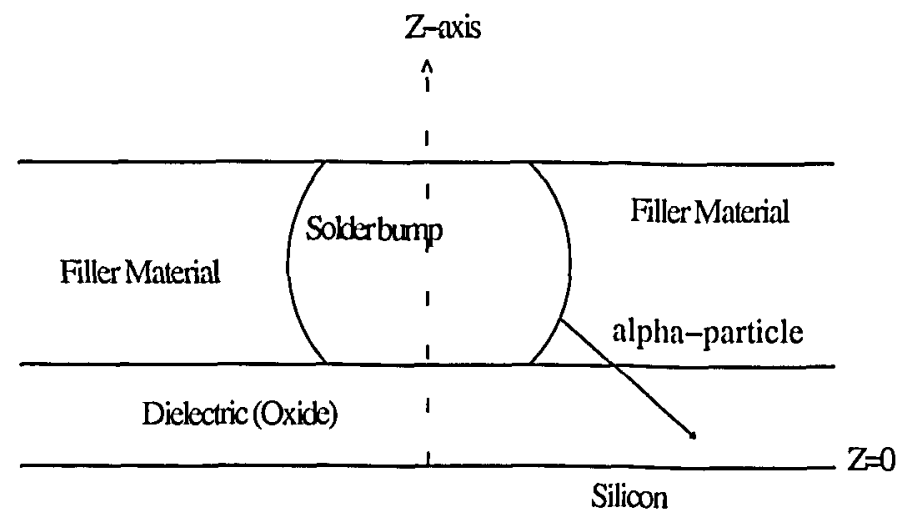

Figure 2: Basic geometry used in calculating the effects of $\alpha$-particles emitted from solder burnps.

simulation does not use monte carlo techniques to estimate soft error rate. A direct integration of the point burst rate and amount of charge needed to cause an upset is coupled with point burst probabilities to obtain the soft error rate estimate.

\section{Summary}

Several simulators were described that constitute a system for determining the effects of energetic particles on circuits. The simulators use information about the doping profiles, the geometry of the circuit being analyzed, properties of underfill and various Inaterials used in back end processing and have been shown shown to match well with measurements. For the first time, simulations of the effects of $\alpha$-particles emitted from solder burnps are shown.

\section{References}

[1] T.C. May and M.H. Woods, "Alpha-Particle Induced Soft Errors in Dynamic Memories," IEEE TED, vol. ED-26, no. 1, pp. 2-9, Jan. 1979.

[2] G.R. Srinivasan et al, "Accurate, Predictive Modeling of Soft Error Rate Due to Cosmic Rays and Chip Alpha Radiation," Proc. IRPS, pp. 12-16, Apr. 1994.

[3] See the entire issue of the IBM Journ. R\&D, vol. 40, no. 1, Jan. 1996.

[4] A. Ditali and Z. Hasnain, "Monitoring Alpha Particle Source During Wafer Processing," Semiconductor International, pp. 136-140, Jume 1993.

[5] W.R. McKee et al., "Cosmic Ray Neutron Induced Upsets as a Major Contributor to the Soft Error Rate of Current and Future Generation DRAMs," Proc. IRPS, pp. 1-6, Apr. 1996.

[6] P. Oldiges, "Alpha-Particle Induced Soft Error Rate Evaluation Tool and User Interface." Simulation of Semiconductor Devices and Processes, vol. 6, SpringerVerlag, pp. 310-313, Sept. 1995. 


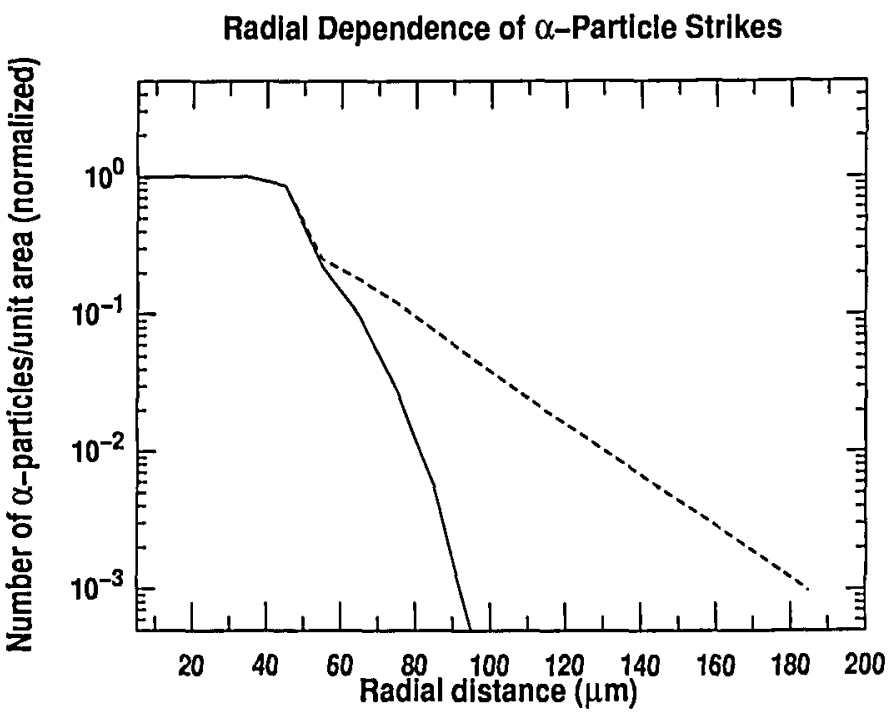

Includling solder ball undertill

.... No underilli materlal

Figure 3: Estimated $\alpha$-particle radial flux distribution at the silicon surface directly under a solder bump.

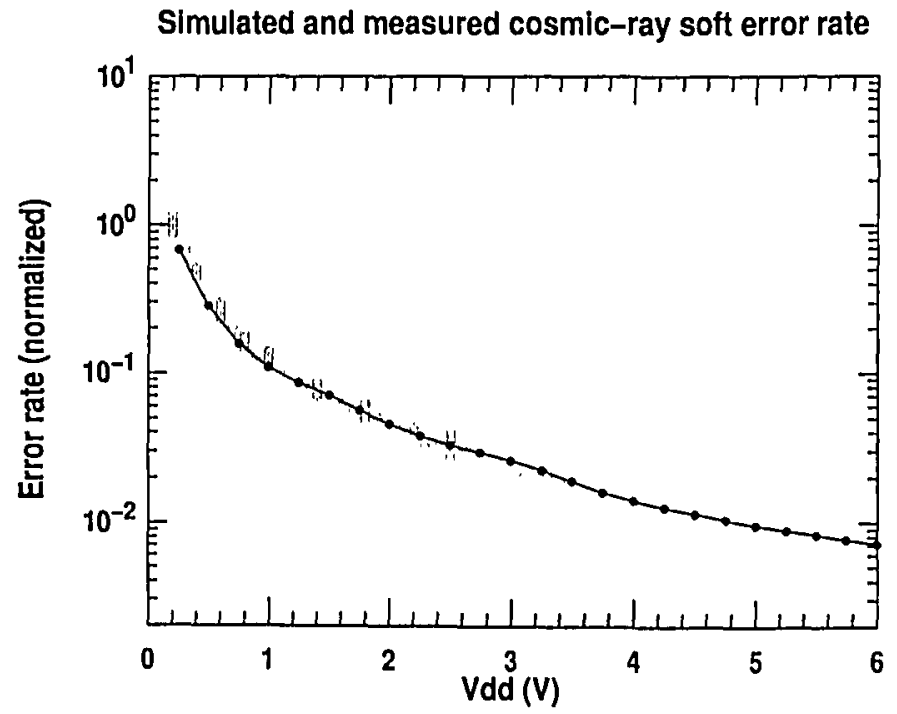

Measurement

Simulatlon

Figure 4: Neutron induced soft error rate. 